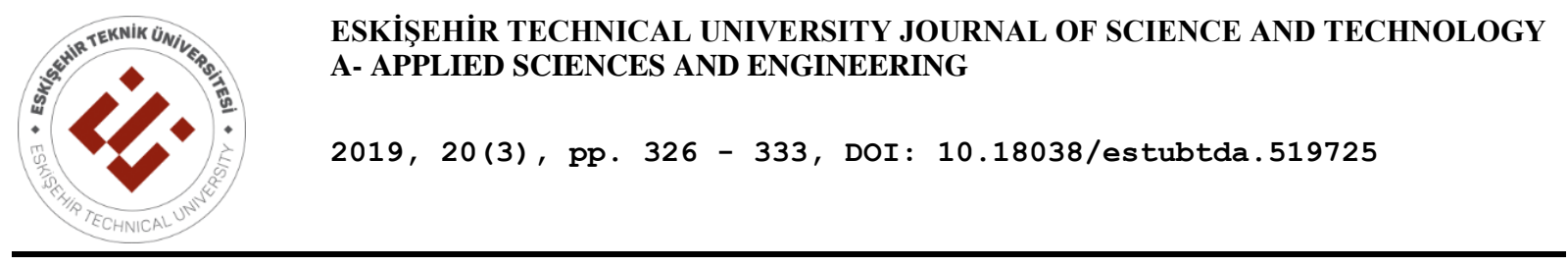

\title{
DYNAMICS OF BOSE EINSTEIN CONDENSATE IN FOURIER SYNTHESIZED OPTICAL LATTICE POTENTIAL
}

\author{
Züleyha ÖZTAŞ* \\ Physics Department, Science Faculty, Eskişehir Thechnical University, Eskişehir, Turkey
}

\begin{abstract}
In this study, we examine the dynamics of Bose Einstein condensate trapped by Fourier synthesized optical lattice potential. We use time-dependent variational method to find the ordinary differential equations of motion. We also solve Gross Pitaevskii equation numerically using split step Fourier method to verify our findings. Good agreement is achieved between the analytical and numerical results.
\end{abstract}

Keywords: Gross Pitaevskii equation, Variational method

\section{INTRODUCTION}

Bose Einstein condensate (BEC) is a state in which a large fraction of bosons exists in the ground state at a temperature nearly absolute zero $[1,2]$. BEC has attracted great interest since it was achieved experimentally [3]. This achievement motivated many experimental and theoretical studies related with the dynamics, excitation spectrum and modulational instability of condensate [4-7]. The dynamics of BEC is theoretically defined by Gross Pitaevskii (GP) equation which is based on the mean field theory. GP equation is a kind of nonlinear Schrödinger (NLS) equation including a trapping potential term and a nonlinear term which describes inter-atomic interactions. The dynamics of condensate changes substantially depending on these two terms.

Variational method is frequently used for problems in BEC and nonlinear optics. This method provides a set of ordinary differential equations describing the dynamics of system approximately. It can be used in BEC studies with several external potentials including harmonic potentials, optical lattice (OL) or time modulated potentials [4-17]. It is well known that the collapse of condensate occurs when the atom number exists a critical value in the attractive nonlinearity regime. It has been found that the variational method, using Gaussian ansatz, gives good predictions for the stability analysis and collapse in the presence of harmonic potential [4-7]. The dynamics of BEC in optical lattices has been investigated using the variational method and instability conditions have been discussed [8-11]. According to these references, the variational approach is good at estimating the critical number of atoms before collapse. Modulational instability analysis has been done for one-dimensional OL by taking higher order interactions into account [11]. Matter wave solutions like bright and dark solitons have been also investigated by the variational method [12-15]. Dynamics in disordered or random potentials is another topic of interest. There are several studies related with Gaussian or soliton variational ansatz in the presence of these kind of potentials [16-17].

In this study, we examine the dynamics of BEC in the presence of Fourier synthesized optical lattice potential. This potential is a kind of ratchet potential which changes with time sinusoidally. The formation of the potential in BEC experiments has been given in [18-19]. In these references, the stationary form of Fourier synthesized OL potential with two components has been considered. The potential has the form $V(x)=V_{1} \cos (2 x)+V_{2} \cos (4 x+\alpha)$. This kind of OL can be achieved by

*Corresponding Author: zdonmez@eskisehir.edu.tr

Received: 30.01.2019 Published: 26.09.2019 
superimposing two lattice potentials which have the phase difference $\alpha$. The first lattice whose depth is $V_{1}$ is a usual standing wave potential while the second lattice related with the potential depth $V_{2}$ is multiphoton potential. These potential depths are connected with the atomic recoil energy and has a role in determining the size of the band gap [18, 19]. The analytical consideration of nonautonomous NLS in Fourier synthesized OL has been done, and bright soliton solutions have been achieved in [20]. The aim of this paper is to investigate the time evolution of BEC under this potential. The paper is organized in this way: We give the theoretical background of BEC in section 2. The solution of the GP equation including Fourier synthesized OL potential is carried out by both the variational and numerical methods. In the last section, we will sum up the results we get in this paper.

\section{THEORETICAL MODEL}

The dynamics of BEC is governed by the GP equation. The dimensionless form of the GP equation in the mean field approach is given by

$$
i \frac{\partial \psi(x, t)}{\partial t}=-\frac{1}{2} \frac{\partial^{2} \psi(x, t)}{\partial x}+V(x, t) \psi(x, t)+g|\psi(x, t)|^{2} \psi(x, t)
$$

where $\psi(x, t)$ is the wave function, and $\mathrm{t}$ and $\mathrm{x}$ are in units of the trapping frequency, $1 / \omega_{\perp}$, and the harmonic oscillator length, $a_{0}=\sqrt{\hbar / m \omega_{\perp}}$, respectively. The nonlinear coefficient $g=4 \pi \hbar^{2} a / m$ determines inter-atomic interactions and is proportional to the scattering length $a$. We assume $g=1$ considering the interactions are repulsive. The trapping potential, $V(x, t)$, is taken as Fourier synthesized OL potential which is given by [20]

$$
V(x, t)=V_{0} c(t)\left[V_{1}+V_{2} \cos (x)+V_{3} \cos (2 x)\right]
$$

where the time dependent part is $c(t)=\sin (\omega t)+\sin (2 \omega t)$, and the other constants in the space dependent part are $V_{1}=24 V_{0}, V_{2}=4 f, V_{3}=8 V_{0}$. Here $f$ is a positive constant, and $V_{0}$, which depends on the intensity of the laser generating the $\mathrm{OL}$, defines the depth of the potential. While $V_{1}$ is a constant, $V_{2}$ and $V_{3}$ determine the depths of the lattice potential components separately. $f$ is an important constant

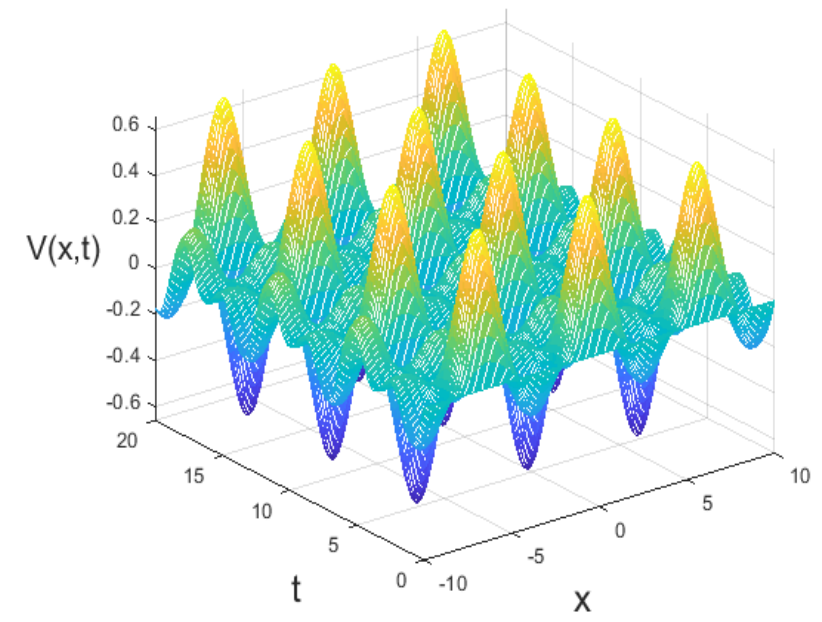

Figure 1: Fourier synthesized optical lattice potential plot for parameters $\omega=1, f=1$ and $V_{0}=1 / 16$.

specifying the depth of the tirst lattice component, $V_{2}$. For the values $f \gg V_{0}, V_{2}$ is very large compared with $V_{3}$ such that $V_{2} \gg V_{3}$. In this case, the multi-photon contribution is a small perturbation term, and 
the potential is shallow. The plot of the potential is given in Figure 1 with the parameters $\omega=1, f=1$ and $V_{0}=1 / 16$. The deep of the potential increases with $V_{0}$, while its frequency is determined by $\omega$.

\subsection{Variational Method}

Variational approach is a good tool to investigate the dynamics of condensate in the presence of both time dependent and time independent potentials. The Lagrangian density of BEC is given by $[4,5]$

$$
\mathcal{L}=\frac{i}{2}\left(\psi_{t} \psi^{*}-\psi_{t}^{*} \psi\right)-\left|\psi_{x}\right|^{2}-V|\psi|^{2}-\frac{g}{2}|\psi|^{4}
$$

We choose a Gaussian ansatz as the trial wave function [21]

$$
\psi(x, t)=A(t) \exp \left[-\frac{x^{2}}{2 a^{2}(t)}+i b(t) x^{2}\right]
$$

The time dependent parameters $A(t), a(t)$ and $b(t)$ are the amplitude, radial width and radial chirping parameters respectively. We ignore the phase of the condensate for the time being. After inserting the ansatz Eq.(4) into Eq.(3), the Lagrangian of the system can be derived via the integration $L=\int_{-\infty}^{\infty} \mathcal{L} d x$. The set of variational parameters is $q=\left(A(t), A^{*}(t), a(t), b(t)\right)$. Taking $c(t)=1, f=1$ and using Euler-Lagrange equation given by

$$
\frac{d}{d t} \frac{\partial \mathcal{L}}{\partial \dot{q}}-\frac{\partial \mathcal{L}}{\partial q}=0
$$

we get the following equations for each variational parameters

$$
\begin{aligned}
& i a \dot{A}^{*}+i \frac{d}{d t}\left(a A^{*}\right)+a^{3} \dot{b} A^{*}+\frac{1}{2}\left(\frac{1}{a}+4 b^{2} a^{3}\right) A^{*}+\sqrt{2} a g|A|^{2} A^{*}+a V A^{*}=0 \\
& i a \dot{A}+i \frac{d}{d t}(a A)-a^{3} \dot{b} A-\frac{1}{2}\left(\frac{1}{a}+4 b^{2} a^{3}\right) A-\sqrt{2} a g|A|^{2} A-a V A=0 \\
& i\left(A^{*} \dot{A}-A \dot{A}^{*}\right)-3 a^{2} \dot{b}|A|^{2}-\frac{4 c}{a^{2}}|A|^{2}-\frac{3}{2}\left(\frac{1}{a^{2}}+4 b^{2} a^{2}\right)|A|^{2}-\frac{\sqrt{2}}{2} g|A|^{4}- \\
& 24 V_{0}^{2}|A|^{2}+2 V_{0} e^{-\frac{a^{2}}{4}}\left(a^{2}-2\right)|A|^{2}+8 V_{0}^{2} e^{-a^{2}}\left(2 a^{2}-1\right)|A|^{2}=0 \\
& \frac{d}{d t}\left(a^{3}|A|^{2}\right)-4 b a^{3}|A|^{2}=0
\end{aligned}
$$

Using Eq.(6) and Eq.(7), we find the following equation

$$
\frac{d}{d t}\left(a|A|^{2}\right)=0
$$

It can be concluded from Eq.(10) that $a|A|^{2}=E$ is an energy like constant of motion and conserved. Combining Eq. (6), Eq. (7) and Eq. (8) gives the time derivative of the chirping constant

$$
a^{2} \dot{b}-\frac{1}{2 a^{2}}+2 b^{2} a^{2}-\frac{1}{2 \sqrt{2}} g|A|^{2}-V_{0} a^{2} e^{-\frac{a^{2}}{4}}\left(1+8 V_{0} e^{-\frac{3 a^{2}}{4}}\right)=0
$$

The chirping parameter can be directly obtained from Eq. (9):

$$
b-\frac{\dot{a}}{2 a}=0
$$

Writing Eq.(12) and its derivative in Eq.(11) gives the second order ordinary differential equation related with the condensate width 


$$
\ddot{a}-a^{-3}-\sqrt{\frac{2}{\pi}} \frac{g E}{2 a^{2}}-2 V_{0} a e^{-\frac{a^{2}}{4}}\left(1+8 V_{0} e^{-\frac{3 a^{2}}{4}}\right)=0
$$

This equation demonstrates the time evolution of the condensate width when the condensate is trapped by Fourier synthesized OL potential. Now we can define the phase $\varphi$ of the amplitude $A$ such that $A=$ $|A| e^{i \varphi}$. After some algebra, one can find from Eq.(6) and Eq.(7)

$$
a^{2} \dot{b}+2 \dot{\varphi}+\frac{1}{2}\left(\frac{1}{a^{2}}+4 b^{2} a^{2}\right)+\sqrt{2} g|A|^{2}+V=0
$$

Substituting Eq.(14) into Eq.(11), we get the equation of motion related to the phase

$$
\dot{\varphi}+\frac{1}{2 a^{2}}+\sqrt{\frac{2}{\pi}} \frac{5 g E}{8 a}+\frac{V^{\prime}}{2}=0
$$

where $V^{\prime}=24 V_{0}^{2}+V_{0} e^{-\frac{a^{2}}{4}}\left(a^{2}+4\right)+8 V_{0}^{2} e^{-a^{2}}\left(a^{2}+1\right)$. Eq.(12), Eq.(13) and Eq.(15) are the set of differential equations which determines the dynamics of the condensate. Eq.(12) and Eq.(15) can be easily obtained after Eq. (13) is numerically solved. In the following subsection, the numerical solution of Eq. (13) is taken into account.

\subsection{Numerical Method}

In this section, we make comparision between the results of the variational and numerical methods. We use MATLAB program to obtain the solutions. Firstly, we numerically solve the ordinary differential equation given by Eq.(13) by the fourth order Runge Kutta method. Secondly, we directly employ split step Fourier method (SSFM) to Eq.(1). SSFM is applied to the GP equation by splitting it into linear and nonlinear parts. The first term in Eq. (1) on the right hand side forms the linear part while the other two terms are nonlinear part. The implementation of SSFM to the GP equation is given by the following equations [22]:

$$
\begin{aligned}
& \psi\left(x, t_{0}+d t\right)=e^{\left(-\frac{i}{2}\left(V+g|\bar{\psi}|^{2}\right) d t\right)} F^{-1}\left[e^{A} F\left[e^{B} \psi\left(x, t_{0}\right)\right]\right] \\
& \bar{\psi}=F^{-1}\left[e^{A} F\left[e^{B} \psi\right]\right]
\end{aligned}
$$

where $A=-\frac{i}{2} k^{2} d t, B=-\frac{i}{2}\left(V+g|\psi|^{2}\right) d t$. Here $F$ indicates the Fourier transform, $k$ is the wave number, $d t$ is the time step, and $t_{0}$ is the initial time. The time evolution of the wave function is found via Eq. (16).We take the time step $d t=0.001$, the space step $d x=0.05$ and Eq.(4) as the initial wave function. The density of the solution of Eq.(1) at time $t=10$ is plotted in Figure 2 for four cases. Figure 2.a is plotted for the case without any potential. We increase the depth of the potential in other three cases. The straigth line shows the results of the numerical solution. The dashed line indicates the results obtained by the variational method. Comparing the predictions of the variational method and the numerical results, we can say that good agreement is achieved between the methods. The amplitude of the wave function at the center decreases with increasing $V_{0}$. Increasing $V_{0}$ further, as in Figure 2.d, we find by the numerical solution that the Gaussian form of the solution begins to destroy. The further increment of $V_{0}$ increases this deterioration. This situation cannot be predicted by the variational approach. In this case, the variational approach is far from exactly describing the central part since it is considered to be valid for Gaussian solutions. Therefore, there is a little discrepancy between the numerical and variational results in Figure 2-d. 


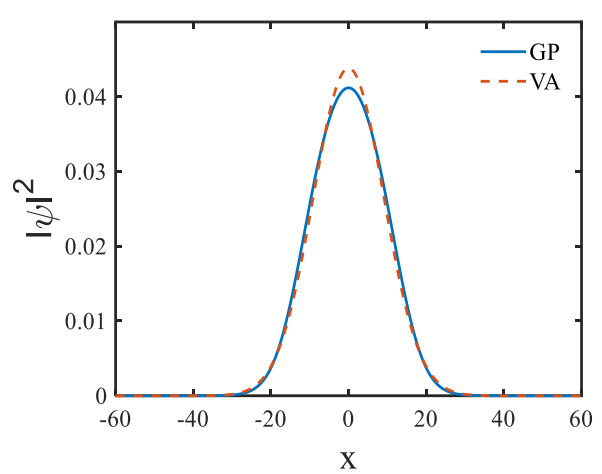

(a)

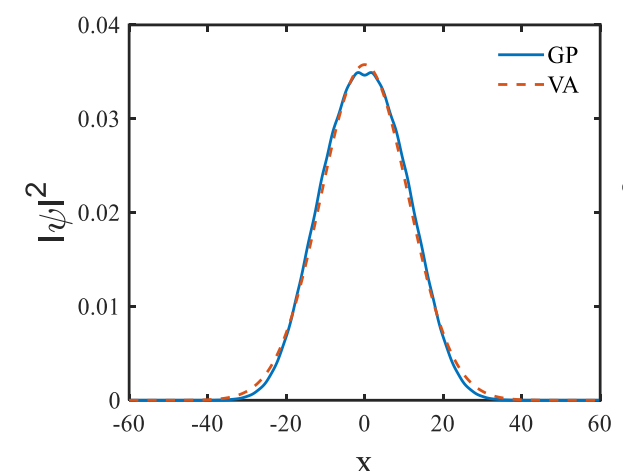

(c)

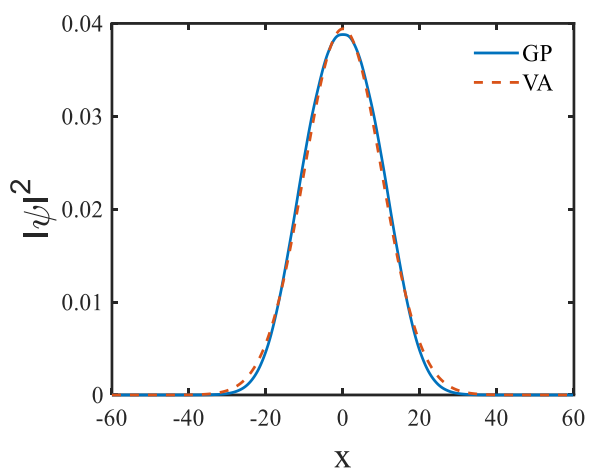

(b)

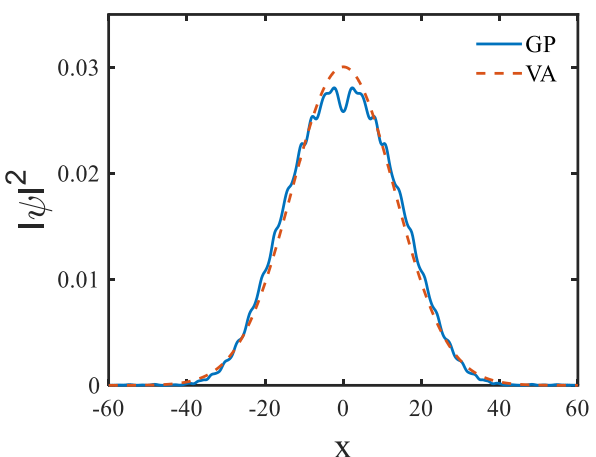

(d)

Figure 2: The density of the condensate with the parameters $\omega=10$, and (a) $V_{0}=0$, (b) $V_{0}=1 / 16$, (c) $V_{0}=$ $2 / 16$, (d) $V_{0}=4 / 16$. The straigth line plots the numerical results while the dashed line indicates the variational method.

Now we can calculate the width and the amplitude separately. Using Eq.(4), it can be easily noticed that the width and the amplitude of the condensate can be achieved numerically from the wave function by the following equations:

$$
\begin{aligned}
& \int_{-\infty}^{\infty}|\psi|^{2} d x=\sqrt{\pi} a A \\
& <|\psi|>=\frac{\int_{-\infty}^{\infty}|\psi|^{3} d x}{\int_{-\infty}^{\infty}|\psi|^{2} d x}=\sqrt{\frac{2}{3}} A
\end{aligned}
$$

After the wave function is obtained by SSFM, $a$ and $A$ can be obtained by Eq.(18) and Eq.(19). The results are represented in Figure 3. Figure 3.a and Figure 3.b show the time evolution the condensate width and the amplitude, respectively. $V_{0}$ is increased from top to bottom. From Figure 3, one can see that the condensate width increases with $V_{0}$ while the amplitude decreases slightly with $V_{0}$. In the case of the vanishing potential, the two lines exactly overlap both for $a$ and $A$. In other cases with non-zero potential, there is a little discrepancy between the lines for $a$ in long time period as can be seen from Figure 3.a. The situation seems to be different for $A$ (Figure 3.b). The variational amplitude is slightly larger than the numerical amplitude in the small time region. However, two methods exactly give the same results with increasing time. Nevertheless, we can conclude that the variational and numerical results are in good agreement with each other. The amplitude decreases smoothly with time for all $V_{0}$ values. 

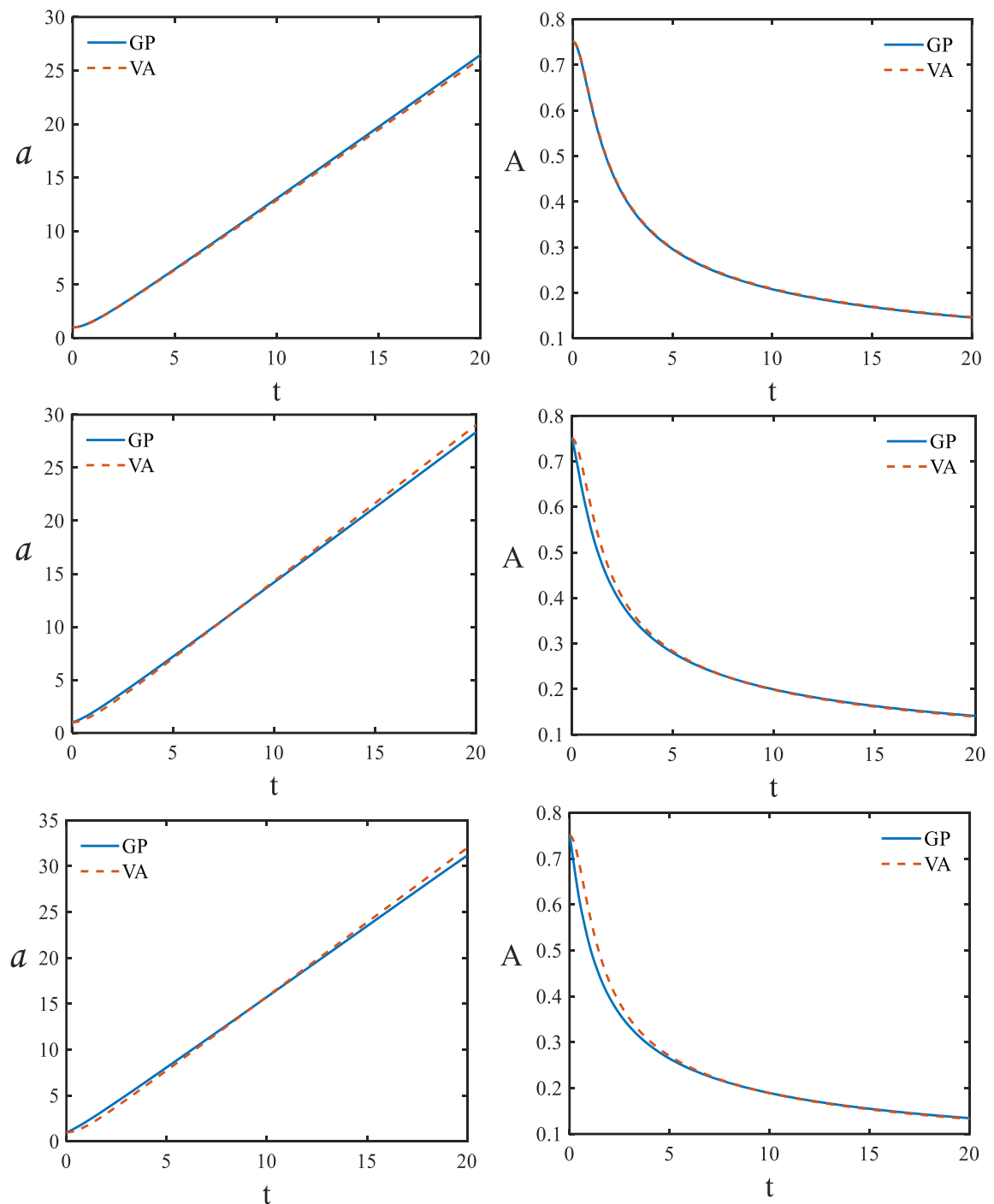

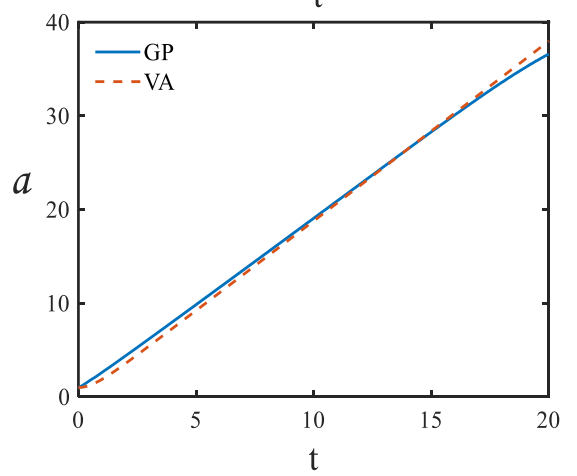

(a)

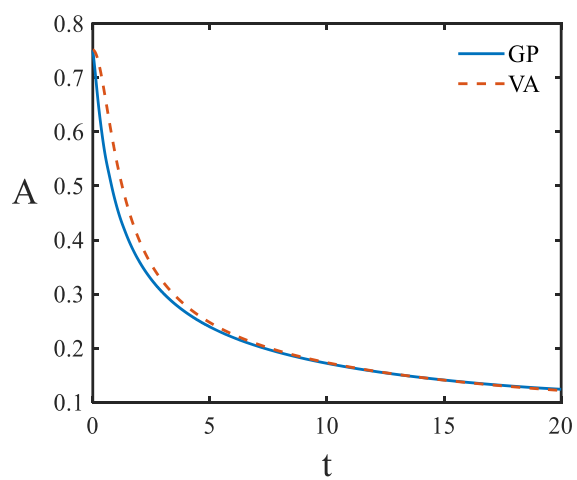

(b)

Figure 3. (a) The width of the condensate versus time. (b) The amplitude of the condensate versus time. The parameters are $V_{0}=0, V_{0}=1 / 16, V_{0}=2 / 16, V_{0}=4 / 16$ from top to bottom, and $\omega=10$. The straigth line plots the numerical results while the dashed line indicates the variational results. 


\section{CONCLUSIONS}

In this paper, we theoretically investigated the dynamics of BEC in the presence of an external Fourier synthesized optical lattice potential. We obtained the equations of motion by the variational method starting from a Gaussian ansatz. The dependence of dynamics on the potential depth has been investigated. We also used split step Fourier method to examine the dynamics of the system. We observed that the results achieved from both methods are generally in accordance with each other. Although some forms of Fourier synthesized OL potential have been studied in BEC experiments [1820], the dynamics of BEC has not been investigated yet in detail. We hope that this work contribute to both experimental and theoretical studies in this field. This work can also be extended to other similar systems such as optical fibers.

\section{REFERENCES}

[1] Bose S N. Plancks Gesetz und Lichtquantenhypothese. Zeitschrift für Physik 1924; 26: 178-181.

[2] Pethick C J, Smith H. Bose-Einstein Condensation in Dilute Gases. United Kingdom: Cambridge University Press, 2002.

[3] Anderson M H, Ensher J R, Matthews M R, Wiemann C E, Cornell E A. Observation of BoseEinstein condensation in a dilute atomic vapor. Science 1995; 269: 198-201.

[4] Perez-Garcia V M, Michinel H, Cirac J I, Lewenstein M, Zoller P. Dynamics of Bose-Einstein condensates: Variational solutions of the Gross-Pitaevskii equations. Phys. Rev. A 1997; 56: 14241432 .

[5] Perez-Garcia V M, Michinel H, Cirac J I, Lewenstein M, Zoller P. Low Energy Excitations of a Bose-Einstein Condensate: A Time-Dependent Variational Analysis. Phys. Rev. Lett. 1996; 77: 5320-5323.

[6] Abdullaev F K, Gammal A, Tomio L, Frederico T. Stability of trapped Bose-Einstein condensates. Phys. Rev. A 2001; 63: 043604-15.

[7] Wamba E, Sabari S, Porsezian K, Mohamadou A, Kofane T C. Dynamical instability of a BoseEinstein condensate with higher-order interactions in an optical potential through a variational approach. Phys. Rev. E 2014; 89: 052917-13.

[8] Burlak G, Malomed B A. Dynamics of matter-wave solitons in a time-modulated two-dimensional optical lattice. Phys. Rev. A 2008; 77: 053606-22.

[9] Cheng Y, Adhikari S K. Spatially antisymmetric localization of matter wave in a bichromatic optical lattice. Laser Phys. Lett. 2010; 7: 824-830.

[10] Sakhel A R. Long-time averaged dynamics of a Bose-Einstein condensate in a bichromatic optical lattice with external harmonic confinement. Physica B: Condensed Matter 2016; 493: 72-80.

[11] Wamba E, Sabari S, Porsezian K, Mohamadou A, Kofane T C. A variational approach to the modulational-oscillatory instability of Bose Einstein condensate in an optical potential. Phys. Lett. A $2013 ; 377: 2408-2415$.

[12] Umarovi B A, Messikh A, Regaai N, Baizakov B B. Variational analysis of soliton scattering by external potentials. Journal of Physics: Conference Series 2013; 435: 012024-9. 
Öztaş / Eskişehir Tech. Univ. J. of Sci. and Technology A-Appl. Sci. and Eng. 20 (3) - 2019

[13] Castro C J, Urzagasti D. Seesaw drift of bright solitons of the nonlinear Schrödinger equation with a periodic potential. Journal of Nonlinear Optical Physics \& Materials 2016; 25: 1650038-8.

[14] Cheng Y. Effective potential of two coupled binary matter wave bright solitons with spatially modulated nonlinearity. J. Phys. B: At. Mol. Opt. Phys. 2009; 42: 205005-8.

[15] Abdullaev F K,Gammal A,Tomio L. Dynamics of bright matter-wave solitons in a Bose-Einstein condensate with inhomogeneous scattering length. J. Phys. B: At. Mol. Opt. Phys. 2004; 37: 635651.

[16] Falco G M. Variational approach for Bose-Einstein condensates in strongly disordered traps. J. Phys. B: At. Mol. Opt. Phys. 2009; 42: 215303-8.

[17] Cheng Y, Adhikari S K. Matter-wave localization in a random potential. Phys. Rev. A 2010; 82: 013631-6.

[18] Ritt G, Geckeler C, Salger T, Cennini G, Weitz M. Fourier synthesis of optical potentials for atomic quantum gases. Phys. Rev. A 2006; 74: 063622-11.

[19] Salger T, Geckeler C, Kling S, Weitz M. Atomic Landau-Zener Tunneling in Fourier-Synthesized Optical Lattices. Phys. Rev. Lett. 2007; 99: 190405-4.

[20] He J R, Li H M. Nonautonomous bright matter-wave solitons and soliton collisions in Fouriersynthesized optical lattices. Optics Commun. 2011; 284:3084-3089.

[21] Ali S K, Pal D, Roy S K, Talukdar B. Application of variational calculus to propagation of coupled pulses in optical fibers. Czechoslovak Journal of Physics 2006; 6: 217-228.

[22] Javanainen J, Ruostekoski J. Symbolic calculation in development of algorithms: split-step methods for the Gross-Pitaevskii equation. Journal of Physics A: Mathematical and General 2006; 39: L179-L184. 